University of Rhode Island

DigitalCommons@URI

Open Access Master's Theses

1991

\title{
Comparison of the EEG Alpha Power of Males and Females During an Attention Task
}

James E. Arruda

University of Rhode Island

Follow this and additional works at: https://digitalcommons.uri.edu/theses

\section{Recommended Citation}

Arruda, James E., "Comparison of the EEG Alpha Power of Males and Females During an Attention Task" (1991). Open Access Master's Theses. Paper 1422.

https://digitalcommons.uri.edu/theses/1422

This Thesis is brought to you for free and open access by DigitalCommons@URI. It has been accepted for inclusion in Open Access Master's Theses by an authorized administrator of DigitalCommons@URI. For more information, please contact digitalcommons@etal.uri.edu. 


\section{COMPARISON OF THE EEG ALPHA POWER \\ OF MAIES AND FEMALES \\ DURING AN ATTENTION TASK \\ BY \\ JAMES E. ARRUDA}

A THESIS SUBMITTED IN PARTIAI FULFILLMENT OF THE REQUIREMENTS FOR THE DEGREE OF

MASTER OF ARTS

IN

PSYCHOLOGY

UNIVERSITY OF RHODE ISLAND

1991 


\section{MASTER OF ARTS THESIS}

$\mathrm{OF}$

JAMES E. ARRUDA

APPROVED :

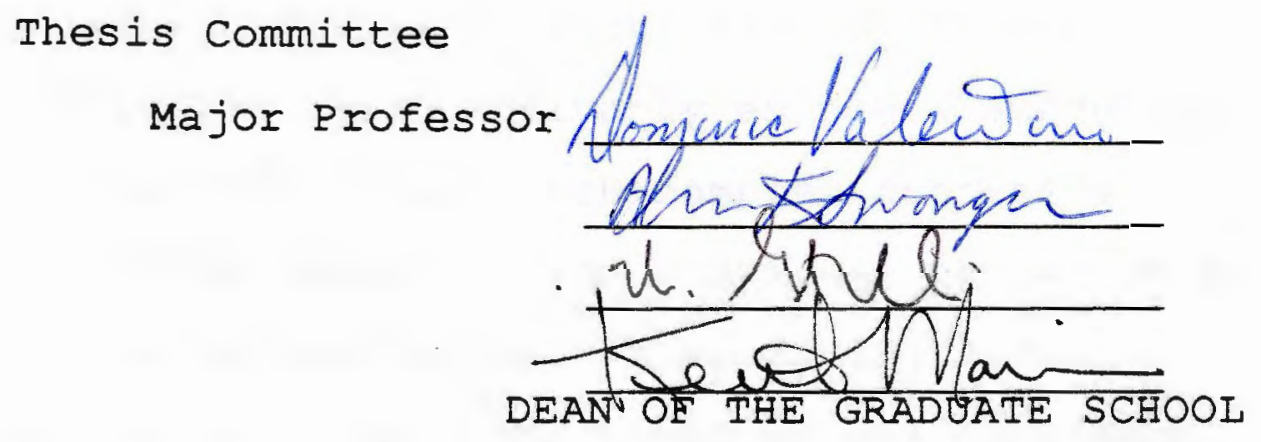

UNIVERSITY OF RHODE ISIAND

1991 


\section{Abstract}

Power spectrum analysis was performed on EEG's from 22 male and 22 female adult subjects under three conditions: 1. Resting; 2. During the first two minutes of the performance of an auditory continuous performance task (CPT); 3. During minutes eight through ten of the performance of an auditory CPT. Studies previously cited in the literature have reported finding electrophysiological gender differences using cognitively complex tasks (e.g. visual and spatial). The successful completion of such complex tasks, however, in no way insures the use of a single cognitive strategy by all subjects. In fact, many different cognitive strategies may conceivably enable a subject to successfully complete a task with complex cognitive dimensions. In the present study a CPT was chosen so as to minimize strategy variation. A mixed ANOVA was performed on the absolute alpha power scores from eight bipolar recording sites. Males and females exhibited comparable lateralization patterns of brain activation during the resting condition and both time periods during the CPT. There was a significant decrease in absolute alpha power in the right temporal-occipital leads and the left temporal-occipital leads for both time periods during the CPT. These data provide evidence that previous observations of gender 
differences during the performance of complex tasks (verbal and spatial tasks) reflect distinct cognitive strategies rather than hard-wiring brain differences. In addition, the data do not support the hypothesis that the right frontal lobe mediates the attention mechanism responsible for maintenance of vigilance. 
Abstract $\ldots \ldots \ldots \ldots \ldots \ldots \ldots \ldots \ldots \ldots \ldots \ldots \ldots \ldots \ldots \ldots \ldots \ldots \ldots$

Table of Contents .......................

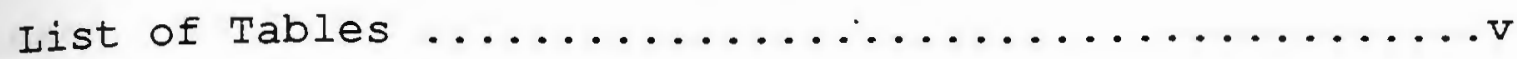

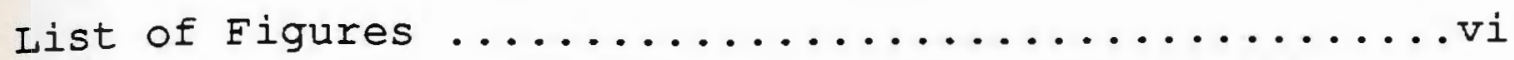
Iiterature Review ........................ 15 Hypothesis and Predictions ............... 15-16 subjects $\ldots \ldots \ldots \ldots \ldots \ldots \ldots \ldots \ldots \ldots \ldots \ldots \ldots \ldots \ldots \ldots \ldots \ldots 17$

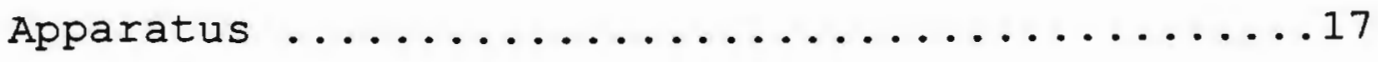
Procedure .........................17-19 Design ............................. $19-20$

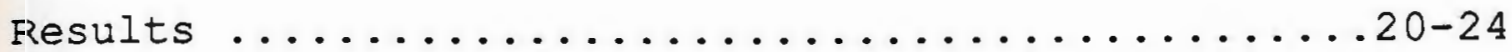

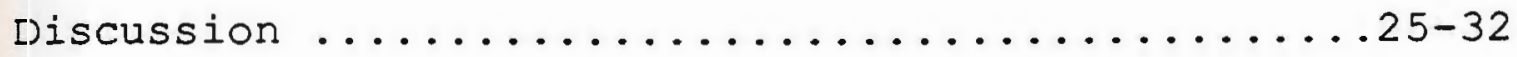

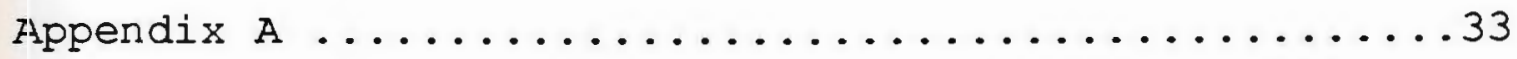

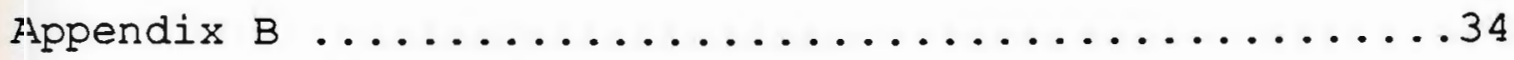

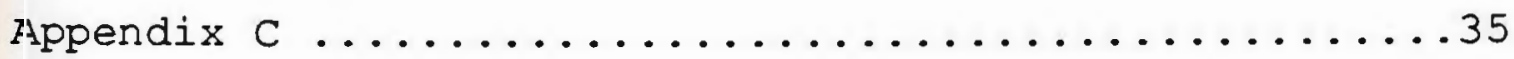

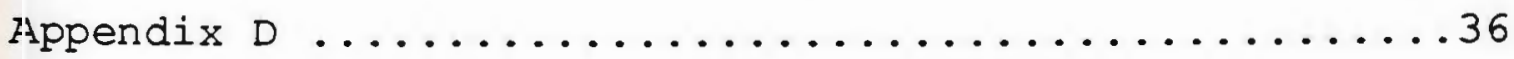

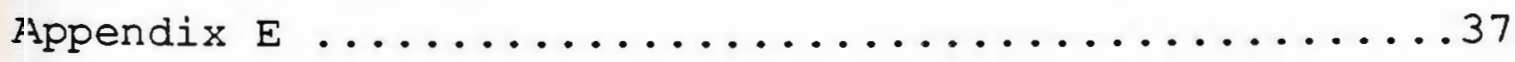

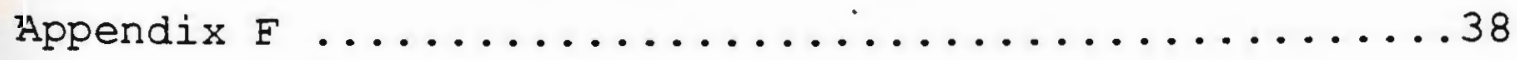
Bibliography ............................ 39-46 
Alpha power means and standard deviations of males and females at the eight bipolar recording sites during

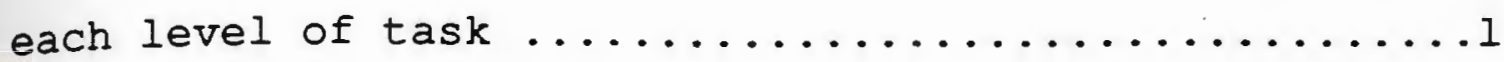

Alpha power means and standard deviations of all subjects at the eight bipolar recording sites during

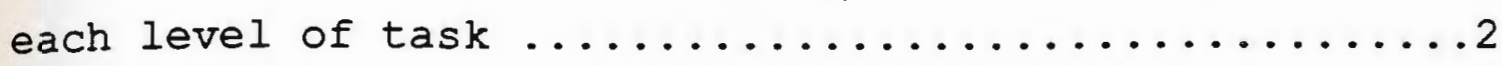

Performance ( $\%$ correct) means and standard deviations of males and females during intake 1

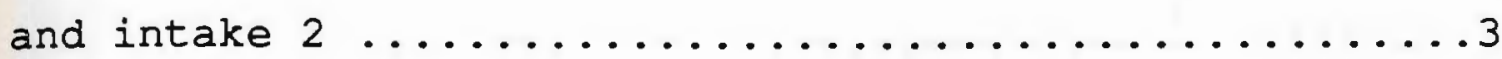


List of Figures

Task by Hemisphere interaction at each region ........ 
One long term goal of our group, The Brain-Behavior Lab, is to establish a set of encephalographic (EEG) norms for "normal", right handed individuals, ages 18 to 25 years, while they perform a simple cognitive task. These norms can then be used to assist physicians and clinicians in their diagnosis of patients with organically based pathologies and may provide a baseline to study the effects of variations in cognitive processes on the EEG. However, the construction of norms is difficult at best and requires that the sample from which the norms were derived be as large and as homogeneous as possible.

Similarly, because the problem of increasing a sample's size is a function of time, the task of making certain that the sample is as homogeneous as possible with respect to all attribute factors (gender, age, and handedness) commands a high priority. Indeed, past research has indicated that the age (Duffy, Albert, McAnulty, \& Garvey, 1984, p. 430) and the handedness (Galin, Ornstein, Herron \& Johnstone, 1982, p.45) of an individual affect that individual's brain physiology and, perhaps, neuroanatomic wiring. Therefore, norms need to be established for each specific sub-populations (i.e. 18 to 25 year old, right handers). The effects of attribute factors other than age and handedness, such as gender, are less understood.

Gender, in particular, may have significant 
influences on an individual's brain structure and physiology. There has been an accumulation of research that has lent support to the theory that the human female brain is more functionally and anatomically symmetric than the human male brain. Consequently, the purpose of this experiment is to investigate whether males and females exhibit dissimilar lateralization of EEG activity while performing a simple attention task. Because the attention task used in this present experiment will be the same task used in the lab's future construction of EEG norms, the results of this experiment will contribute as well to beginning the process of establishing reliable norms.

Sex differences in EEG and in task performance (a behavioral measure of subject vigilance) were analyzed. Because the detection of brain activation was paramount to this investigation, and because "alpha suppression is greater over the active cerebral hemisphere" (Marquis, Glass \& Corlett, 1984, p. 205), the level of alpha power (brain wave ranging from 7.5 to $12.5 \mathrm{hz}$ ) was utilized as an indicator of hemispheric activation/innactivation.

\section{Support for Brain / Gender Hypothesis}

Past neuropsychological research has indicated that anatomical and functional asymmetry differences may exist between the male and the female cerebral hemispheres. Anatomical, clinical, and normative studies have suggested that the female cortex is both 
functionally and anatomically more symmetrical than the male cortex. Hence, it has been postulated that "men and women differ in the degree to which the cerebral hemispheres are specialized for processing different types of information" (Berfield, Ray \& Newcombe, 1986, p. 731).

Anatomical Studies: Recent studies of human and primate brains, using methods ranging from post-mortem examination to magnetic resonance imaging (MRI) and computerized axial tomography (CAT), have provided conflicting results regarding anatomical, gender brain differences. Lacoste-Utamsing and Holloway (1982), after measuring the posterior fifth of nine male and five female corpus callossa post-mortem, reported finding that the splenium was much larger in the female brain than in the male brain (p.1431). In light of the fact that the corpus callosum allows the two hemispheres to communicate, and because females exhibit relatively larger corpus callossa than males, there may exist a greater capacity, and possible need, for the two hemispheres of the female brain to interact. However, Witelson (1985), in an attempt to directly replicate Lacoste-Utamsing and Holloway's previous study using a larger sample size ( 12 male and 30 female), reported finding no significant size differences in the corpus callosum of males and females (p.666). Oppenheim, Lee, Nass, and Gazzaniga (1987), using MRI, studied the 
callossa of 80 living human subjects, and much like Witelson, reported finding no significant differences between males and females regarding callosum width, area, length, or shape (p. 605). In 1988 Lacoste and woodward conducted a post-mortem study on the callossa of 56 primates (four species). The results of this experiment indicated that among the pongids, human kind's closest evolutionary relative, females exhibited significantly greater surface area and splenial width than did males (p.322).

In addition to the corpus callosum, researchers have also been interested in possible cortical differences between males and females. The perceived role of the cerebral hemispheres in human cognition, coupled with the popular belief that the genders were singularly proficient at performing various cognitive tasks (e.g. math and language), fostered this scientific interest. Consequently, the cerebral hemispheres were targeted as areas of the brain that would provide the greatest differentiation of males and females. Wada, Clarke and Hamm (1975), after the post-mortem examination of 100 temporal planums (planum length), found no significant gender differences in planum size. (However, they concluded that there was a trend for the left planum in the male to be larger than the left planum in the female (i.e. $p>.1)(p .243)$ ). In addition, many studies that have examined the length and 
width of male and female hemispheres by CAT have reported finding no significant gender differences (Chui \& Damasio, 1980; Koff, Naeser, Pieniadz, Foundas \& Levine, 1986; Yeo, Turkheimer, Raz \& Bigler, 1987). on the other hand, Bear, Schiff, Saver, Greenberg and Freeman (1986), also using the CAT technique, examined the cerebral hemispheres of 66 subjects and reported finding the male brain to be more asymmetrical with enhanced right-frontal and left-occipital predominance (p.602).

lacking consensus, the results of these anatomical investigations serve to bolster the need for more quality, anatomical research. As a result, no amount of confidence can be had in either of the two hypotheses (i.e. difference vs. no difference).

\section{Clinical studies: Clinical studies, examining} lesion sequelae, have resulted in an even more inconsistent picture of gender brain differences. Lansdell, in the early 1960s, conducted a series of experiments that has lent support to the theory that males were less functionally symmetric than females. As an example, males with left temporal lobe ablation were found to exhibit greater impairment in proverb interpretation (Lansdell, 1961), in word association (Lansdell, 1973), and in performance on the verbal scale of the Wechsler Adult Intelligence Scale (WAIS) (Lansdell, 1968b) than did females. However, Iansdell 
did not find any significant gender differences in the vocabulary performance of subjects with left temporal lobe ablations (Lansdell, 1968a). Lansdell also reported that males with right temporal lobe ablations exhibited decrements in performance on spatial tasks such as the Graves Design Judgment Test (Lansdell, 1962) and the nonverbal (i.e. performance) subtest of the WAIS (Iansdell, 1968b), whereas females did not. Also reported was the fact that males and females performed comparably on the Mooney's Closure Faces Test (Lansdell, 1968a).

From the late 1970 s to the late 1980 s researchers had begun to take seriously the theory that the male brain and the female brain were functionally different. In 1978, McGlone studying the effects of unilateral brain damage $(n=70)$, found that males with left temporal lobe damage earned significantly lower VIQ scores on the WAIS than did their female counterparts. Conversely, MCGlone also discovered that males with right temporal lobe damage earned significantly lower PIQ scores than did females with right temporal lobe damage (p.124). Other researchers have reported comparable results (Edwards, Ellams \& Thompson, 1976; Friedland \& Kershner, 1986; Inglis \& Lawson, 1981; MCGIone, 1984; Sundet, 1986; Yeo, Turkheimer \& Bigler, 1984). Lewis and Kamptner (1987), after examining the performance of 66 unilaterally brain 
damaged subjects on the Street Test (a visual-perceptual gestalt task) and the Block Design Test, concluded that women exhibited "a pattern of scores suggestive of a greater degree of bilateral representation of visuospatial function" (p.148). Inglis and Lawson (1982) in a metaanalysis of 16 clinical studies, totaling 899 subjects, reported that males and females with unilateral brain damage exhibited significantly different PIQ and VIQ scores (P.679). In fact, "greater preponderance of men in either the left or right lesion groups was found to be associated with larger test specific deficits in the verbal and the Performance Scales respectively" (Inglis \& Lawson, 1982, p.670). Bornstein and Matarazzo (1982), in a comparable review of the literature, reported results analogous to Bornstein and Matarazzo's previously cited results.

Other researchers investigating the effects of brain injury, however, have reported finding no significant, functional brain differences between the genders. Bornstein (1984), after studying 32 left hemisphere damaged and 31 right hemisphere damaged subjects with the Revised wechsier Adult Intelligence Scale (WAIS-R), reported finding male and female VIQ and PIQ scores to be quite similar (p.606). Snow and Sheese (1985), after recognizing the interpretation difficulties of Bornstein's WAIS-R results, examined the VIQ and PIQ scores of 35 unilaterally brain damaged 
subjects with the WAIS and also found no significant differences between males and females. In 1986 Herring and Reitan examined the performance of 124 subjects (48 left lesioned; 48 right lesioned; 28 controls) on the Wechsler-Bellevue scale and, similar to the two previously reviewed studies, also failed to find significant VIQ and PIQ differences between males and females.

In reviewing the clinical literature it is apparent that a clear consensus has not been forthcoming. In addition, because of the correlational nature of clinical research (i.e. the location and the size of a subject's lesion lay beyond manipulation) more investigation in this area must be performed if the scientific community is to become confident in the existence of the phenomenon.

EEG Studies: Gender differences in functional brain asymmetry have also been examined with unobtrusive brain imaging techniques (normative research) such as the electroencephalograph (EEG). In 1976, Ray, Morell and Frediani studied the alpha power asymmetry of 12 right handed subjects ( 6 male and 6 female) while they performed six tasks specifically designed to engage either the left or the right hemisphere. The four tasks used to engage the left hemisphere included addition, verb counting, sentence construction, and multiplication. The two right hemisphere tasks were 
music listening, and visualization. Ray et al. reported that females exhibited less hemispheric specificity (i.e. alpha power asymmetry) for the left and right hemisphere tasks than did males (p.393). Wogan, Kaplan, Moore, Epro and Harner (1979), in a similar experiment, examined the alpha asymmetry of 11 right handed subjects ( 6 males and 5 females) while they performed six separate cognitive tasks (resting, vigilance task, mental letter task, block design task, embedded figures task, rod-frame task) and concluded that females were not as consistently lateralized as were males (p.222). Similar results were reported in a study conducted by Trotman and Hammond (1979). After examining the alpha asymmetry of 10 right handed subjects ( 5 male and 5 female) while performing three verbal and three spatial tasks, Trotman and Hammond reported that only males exhibited task-related alpha asymmetries and concluded that such results suggested "a stricter hemispheric lateralization of underlying function in the male brain than in the female brain" (p.430). In 1984, Glass, Butler, and Carter reported a study that examined the alpha asymmetry of 48 human subjects $(24$ male and 24 female) while they performed tasks designed to engage the left and the right hemispheres. Glass et al. "found that the asymmetry of hemispheric activation during mental arithmetic was significantly reversed during the recognition of faces task in males ... but not in 
females" (p.182). Other EEG researchers have reported similar results (Davidson, Schwartz, Pugash \& Bromfield, 1976; Earle \& Pikus, 1982).

However, other researchers have reported results that do not support the gender-brain difference hypothesis. Davidson et al. (1976, p.130), in a builtin replication of their previously cited EEG study, reported finding no significant differences between male and female alpha asymmetry scores $(n=20)$. Similarly, Galin, Ornstein, Herron and Johnstone (1982), after examining the alpha asymmetries of 90 subjects ( 45 male and 45 female) while they performed left and right hemisphere tasks, also reported finding no significant alpha asymmetry differences between males and females (p.49) .

Hence, the normative (EEG). literature has suffered in much the same way as has the anatomical and the clinical literature. The effects of conflicting results has rendered any clear statement of relationship between gender and the brain (i.e. brain physiology, brain anatomy) virtually impossible. Nevertheless, just as the gender-brain difference theory has not enjoyed consistent empirical support, its alternative hypothesis has not fared much better.

\section{Attention and Brain Function}

In addition to examining the impact that the spatial and the verbal components of tasks have on the 
male and female brain, a number of researchers have also examined the influence of attention. As a result, clinical, behavioral, and brain imaging studies (i.e. event related potential studies [ERP], cerebral blood flow studies [CBF], positron emission tomography [PET], and EEG) have constructed an intriguing view of the attending human brain.

Clinical and Behavioral Studies: The accumulation of results obtained from clinical and behavioral attention studies have implicated the right hemisphere as well as the frontal and the parietal lobes in human attention. Rapcsak, Verfaellie, Fleet, and Heilman (1989), after examining the performance of eight subjects with right hemisphere lesions on a visual cancellation task (i.e. an attention task), reported that subjects who had suffered right-frontal brain damage exhibited an "inability to utilize visual selective attention to focus on the critical stimulus variable" (p.181). Unfortunately, gender was not included as a factor in this experiment. In 1982 Salmaso and Denes examined the performance of 20 unilaterally brain damaged subjects (i.e. five rightfrontal lesioned, five left-frontal lesioned, five right-parietal lesioned, and five left-parietal lesioned) on verbal and spatial vigilance tasks and reported that subjects with frontal lesions, either right or left, performed significantly worse than 
subjects with parietal lesions (p.1148). Consequently, Salmaso and Denes concluded that the frontal lobes had participated in the information processing analyses critical to the successful completion of a overtly simple attention task such as the detection of novelty (Salmaso \& Denes, 1982, p.1150). Unfortunately, gender was not included into the design of this investigation, and as a result, the possible effects of gender were overlooked. In 1978 Heilman and Van Den Abell conducted a behavioral study that examined the reaction times of 24 normal right handed subjects $(12$ males and 12 females) while they performed a visual attention task. It was reported that "a warning stimulus presented to the left visual field [of males and females] reduced reaction times more than warning stimuli presented to the right visual field [of males and females]" (Heilman \& Van Den Abell, 1978, p.317). Because stimuli presented in either the left or the right visual field project directly to the right and to the left hemispheres, respectively, the results of this experiment lend support to the theory that the right cerebral hemisphere plays a more important role in attention for both males and females than does the left cerebral hemisphere.

Researchers using brain imaging techniques such as ERP, CBF, PET, and EEG have examined the effects of visual, auditory and tactile vigilance tasks on cerebral 
cortex activation. Together, these brain imaging studies have reported results implicating the right hemisphere, the parietal lobes (left and right), and the frontal lobes (left and right) in attention. As an illustration, Harter, Miller, Price, LaLonde, and Keyes (1990) examined the ERPs of 86 children (51 male and 35 female, ages six to nine) at the frontal, temporal, parietal, central and occipital regions while they performed a visual attention task and reported that the right parietal and the right prefrontal cortex exhibited significantly more activation than did the other cortical areas (p.234). More importantly, Harter et al. reported finding no significant lateralization differences between males and females (1990, p.233). In 1982, Roland examined the cerebral blood flow (CBF) of 10 subjects while they performed an auditory, a visual, and a tactile attention task. For all three modes of attention the right superior frontal cortex, and the right hemisphere in general, exhibited an overall increase in blood flow while other areas of the cerebral cortex did not (p.1075). Once again, the possible effects of gender were not under study. In a related study, Haier, Siegel, Neuchterlein, Hazlett, Wu, Paek, Browning, and Buchsbaum (1988) examined the effects of a visual attention task on the brain metabolism (PET) of 30 male subjects and reported that the right hemisphere exhibited significantly greater activation than did the 
left hemisphere (p.211).

studies of attention have also been conducted with the assistance of EEG. For instance, Ray and Cole (1985), after examining the frontal and the parietal alpha power of 18 right handed subjects $(9$ male and 9 female) while they performed two separate visual attention tasks (i.e. a rejection task and an intake task), reported that the left parietal site exhibited significantly less alpha power activity than did the right parietal site during both attention tasks (p.751). Gender was initially included as a factor within the design of this experiment, however, the effects of gender were not reported. Heilman and Van Den Abell (1980) examined the alpha power of 12 subjects ( 6 male and 6 female) at the frontal, central, parietal and occipital regions while subjects performed a visual attention task and reported finding that whereas the right parietal region desynchronized equally for contralaterally and ipsilaterally presented stimuli, other areas of the cortex, specifically the left parietal region, did not (p.328). This finding would seem to indicate that the right hemisphere might somehow be dominant for attention. In spite of the fact that Heilman and Abell included six males and six females in the study, the effects of gender were not reported. In 1984, Marquis, Glass, and corlett examined the alpha power of 12 subjects ( 6 male and 6 female) at the 
occipital regions while subjects performed a visual attention task. It was reported that the right occipital region exhibited the greatest alpha suppression in relation to the other cortical region (Marquis, Glass \& Corlett, 1984, p.209). The effects of gender were not reported. In 1982 shepherd examined the ffects of gender and attention (i.e. an auditory, vigilance task) on the occipital-parietal alpha power of 40 subjects (20 male and 20 female). Shepherd reported finding no significant difference between the absolute alpha power of males and females or of their left and right hemispheres (1982, p.18).

In summation, the results of numerous clinical, behavioral, ERP, CBE, PET and EEG studies have served only to sketch a picture of the attending, human brain. Nevertheless, it is apparent from the results of these studies that the frontal and the parietal lobes, as well as the right hemisphere in general, play an important role in the cognitive process of attention both for males and for females.

\section{Bypothesis and Prediction}

In the present investigation the effects of gender and attention on cerebral activation (i.e. alpha suppression) was being examined. It was hypothesized that:

1) males and females have differently organized brains; the different functions are shared more by the hemispheres in the female; 
2) alpha power is inversely related to brain activation, and thus, level of attention;

3) there is an attention mechanism, specific to vigilance, in the right, frontal area;

4) the longer a subject is forced to be attentive, the greater the loss of attention becomes.

Corresponding predictions:

1) there will be a task $x$ hemisphere $x$ gender interaction, with only males exhibiting significant differences in alpha power between the left and right hemispheres;

2) for all subjects: alpha power resting $>$ alpha power intake $2>$ alpha power intake 1;

3) all subjects alpha power at the right, frontal leads will be significantly less than at the left, frontal leads during both intake 1 and intake 2;

4) all subjects will perform significantly better (a greater percent correct) during intake 1 than in intake 2 .

\section{Method}

Subjects

Data used in this study comes from a subsample of participants, consisting of forty-four right handed males and females (22 female and 22 male), who participated in an experimental protocol under the supervision of Dr. Dominic valentino. This protocol was reviewed and accepted by the University of Rhode Island Human Subjects Review Board. Subjects were recruited from a general psychology course, PSY 113. Subjects earned credit towards their final course grade in return for their participation. The ages of the 
subjects range from 18 to 25 years.

\section{Apparatus}

An Axon Systems data acquisition system was used to amplify, digitize, and measure subject's brain waves. The recorded measures were subjected to a spectral analysis by Fast Fourier Analysis on an IBM compatible, AT style computer. The CPT task was presented by tape recorder through a speaker directly in front of and above the subject. A paper response recorder (model P2C), manufactured by Ralph Gerbrands Co., was used to record targets and subject responses on response paper.

\section{Procedure}

Participants sat in a comfortable lounge chair while electrodes were placed, according to the International 10-20 system (Jasper, 1958), over the left and right frontal-poles (FP), frontal (F), temporal and occipital (0) areas. Bipolar recording sites were $\mathrm{Fp} 1 / \mathrm{F} 7, \mathrm{Fp} 2 / \mathrm{F} 8, \mathrm{~F} 7 / \mathrm{T} 3, \mathrm{~F} 8 / \mathrm{T} 4, \mathrm{~T} 3 / \mathrm{T} 5, \mathrm{~T} 4 / \mathrm{T} 6, \mathrm{~T} 5 / 01$ and T6/O2 (see Appendix A). A ground electrode was placed in the middle of the forehead.

Participants were given the following instructions:

For the first phase of this experiment we ask that you sit quietly with your eyes closed; your arms in your lap and your legs extended outward. After resting for a period of approximately three minutes we will ask that you perform a task which will constitute phase two: The task will require that you listen to a tape recording in which the letters of the alphabet are spoken randomly, one right after the other. When you hear the same letter spoken twice, (e.g. a b d d k) 
consider it a target (i.e. d d) and press the button (the subject holds a button in his/her right hand). Please keep your eyes closed throughout the procedure (both phases) and try not to move in the seat. We will verbally signal you when we are about to begin phase two.

The participants relaxed for approximately 2 minutes and 45 seconds, while a 2-minute sample of foct iface electroencephalogram was taken. The EEG recording began 45 seconds into the resting period (Resting). After this resting period had ended, participants began performing the continuous performance task for approximately 8 minutes and 45 seconds. Twominute samples of artifact-free electroencephalogram and of performance were taken 45 seconds from the beginning of the tape recording (Intake 1). Six minutes after the commencement of the second two minute EEG sample, an additional two-minute sample of artifact-free electroencephalogram and performance was taken (Intake 2) (see Appendix B).

Both alpha power and performance served as dependent measures in this investigation. Alpha power was measured at each of the eight bipolar channels located laterally around the subject's head during resting, intake 1 and intake 2 (see Appendix A). Performance, ( ( \# of total possible targets - \# of omission errors) / (\# of total possible targets)) $x$ 100), was calculated for each subject, during each of the intake levels only. Because subjects were "resting" 
during the resting level of TASK, a measure of performance was not relevant.

\section{Design}

The following two analyses were performed: 1) a four-waY ANOVA (GENDER $x$ TASK $x$ HEMISPHERE $x$ REGION) waS performed on alpha power scores; 2) a two-way ANOVA (GENDER $x$ TASK) was performed on the subject's performance scores.

Factors of 4 Way ANOVA: There were three levels of TASK (a repeated measures factor), with the amount of subject vigilance being manipulated. HEMISPHERE and REGION were additional repeated measures factors that were incorporated into the design of this experiment. Hemisphere was comprised of two levels (left and right) and region was comprised of four levels (frontal, frontal-temporal, temporal, and temporaloccipital) (see Appendix c). The between subject variable was gender, with males and females making up the two levels. Hence, a randomized block design with repeated measures over TASK, REGION and HEMISPHERE was used in this experiment ( $\mathrm{A} \times(B \times C \times \mathrm{D} \quad \mathrm{S})$ ).

Factors of 2 way ANova: The factors of this design included GENDER (a between groups factor) with two levels and TASK (a repeated measures factor) with only two levels: Intake 1 and Intake 2. Consequently, the design can be symbolized as follows: (A $x$ ( $\mathrm{x}$ S)). 


\section{Results}

EEG Data

The EEG data were first evaluated by a four way ANOVA with GENDER, TASK, HEMISPHERE, and REGION as factors. Mean alpha power and standard deviations for males and females at each of the eight bipolar sites during resting, intake 1 , and intake 2 can be seen in Table 1 .

Prediction \#1: No significant main effects or significant interactions with gender were obtained (see Appendix D). Therefore the data were collapsed over GENDER and an ANOVA was computed using the three remaining repeated measures factors. 
Table 1

Alpha power means and standard deviations for males and females at the elght bipolar recording sites for each level of task

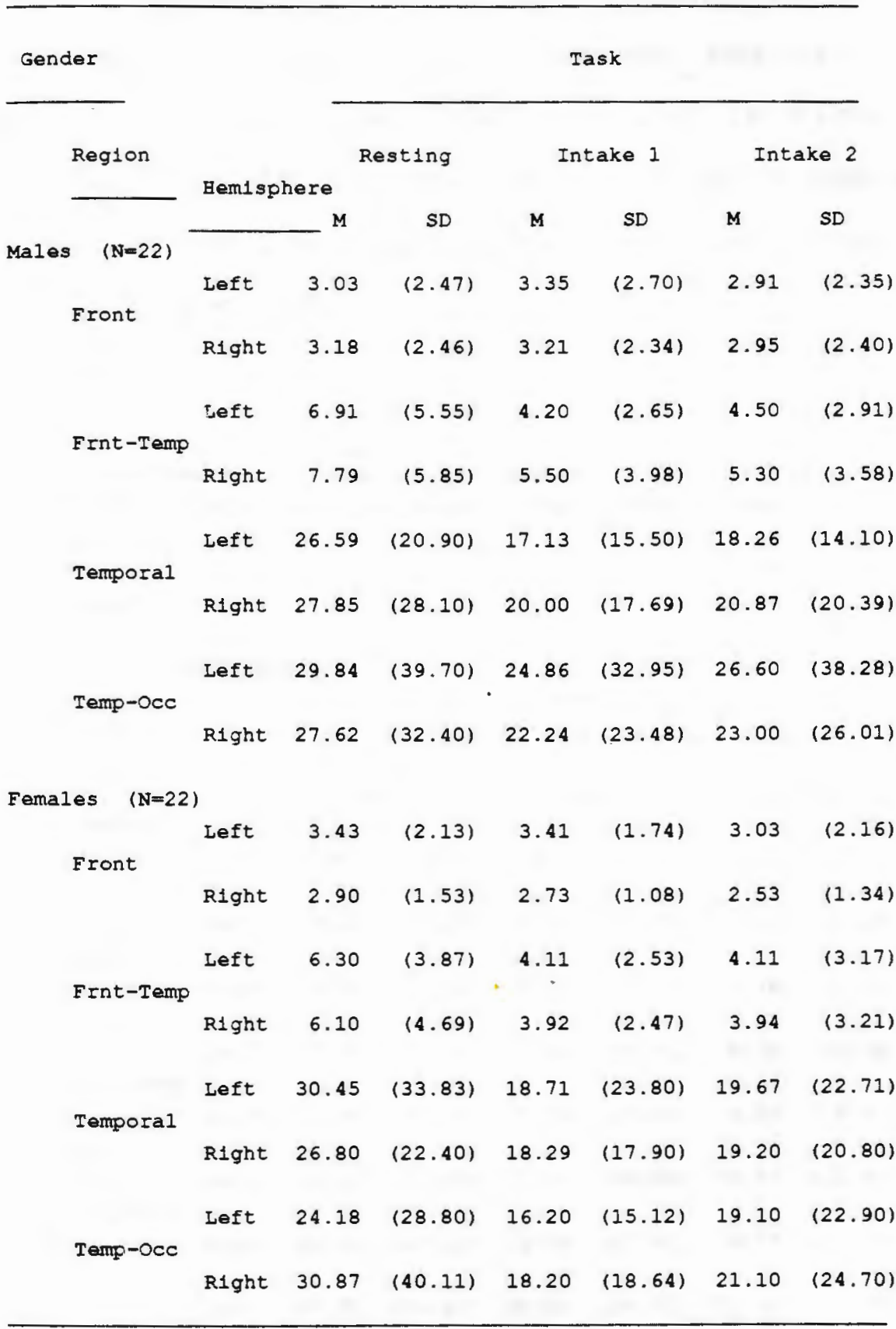

Table 2 shows the mean alpha power and standard deviations for all subjects collapsed over Gender, at each of the eight bipolar recording sites. 
A significant three-way interaction was obtained involving Task, Hemisphere and Region, $F(6,258)=4.31$, $p<.01$, (see Appendix E). The significant three-way interaction was followed up by a two-way analysis ( $\mathrm{x}$ H) for each of the four regions, as shown in Figure 1 . The results of the simple interaction effects tests were only significant at the Temporal-occipital region, $F(2,86)=3.17, P<.05$.

Table 2

Alpha power means and standard devlations for all subjects at each of the elght electrode sltes for each level of task

Reg1on

\begin{tabular}{|c|c|c|c|c|c|c|c|}
\hline \multirow{3}{*}{$(N=44)$} & & \multicolumn{6}{|c|}{ Task } \\
\hline & & \multicolumn{2}{|c|}{ Rest1ng } & \multicolumn{2}{|c|}{ Intake 1} & \multicolumn{2}{|c|}{ Intake 2} \\
\hline & & $\mathbf{M}$ & $S D$ & $\mathbf{M}$ & SD & $\mathbf{M}$ & $S D$ \\
\hline & Left & 3.37 & $(2.28)$ & 3.38 & $(2.25)$ & 2.97 & $(2.23)$ \\
\hline \multicolumn{8}{|l|}{ Front } \\
\hline & RIght & 3.04 & $(2.03)$ & 2.97 & $(1.82)$ & 2.74 & (1.93) \\
\hline & Left & 6.63 & $(4.74)$ & 4.16 & $(2.56)$ & 4.31 & $(3.01)$ \\
\hline \multicolumn{8}{|c|}{ Frnt-Temp } \\
\hline & R1ght & 6.94 & $(5.31)$ & 4.72 & $(3.36)$ & 4.66 & $(3.44)$ \\
\hline & Left & 28.52 & $(27.87)$ & 17.92 & (19.88) & 18.97 & $(18.69)$ \\
\hline \multicolumn{8}{|l|}{ Temporal } \\
\hline & R1ght & 27.33 & $(25.13)$ & 19.14 & $(17.62)$ & 20.03 & $(20.38)$ \\
\hline & Left & 27.01 & $(34.41)$ & 20.53 & $(25.71)$ & 22.85 & $(31.40)$ \\
\hline \multicolumn{8}{|l|}{ Temp-Occ } \\
\hline & R1ght & 29.25 & $(36.06)$ & 20.23 & $(21.05)$ & 22.05 & $(25.09)$ \\
\hline
\end{tabular}


Figure 1. Task by Hemisphere interaction at each region
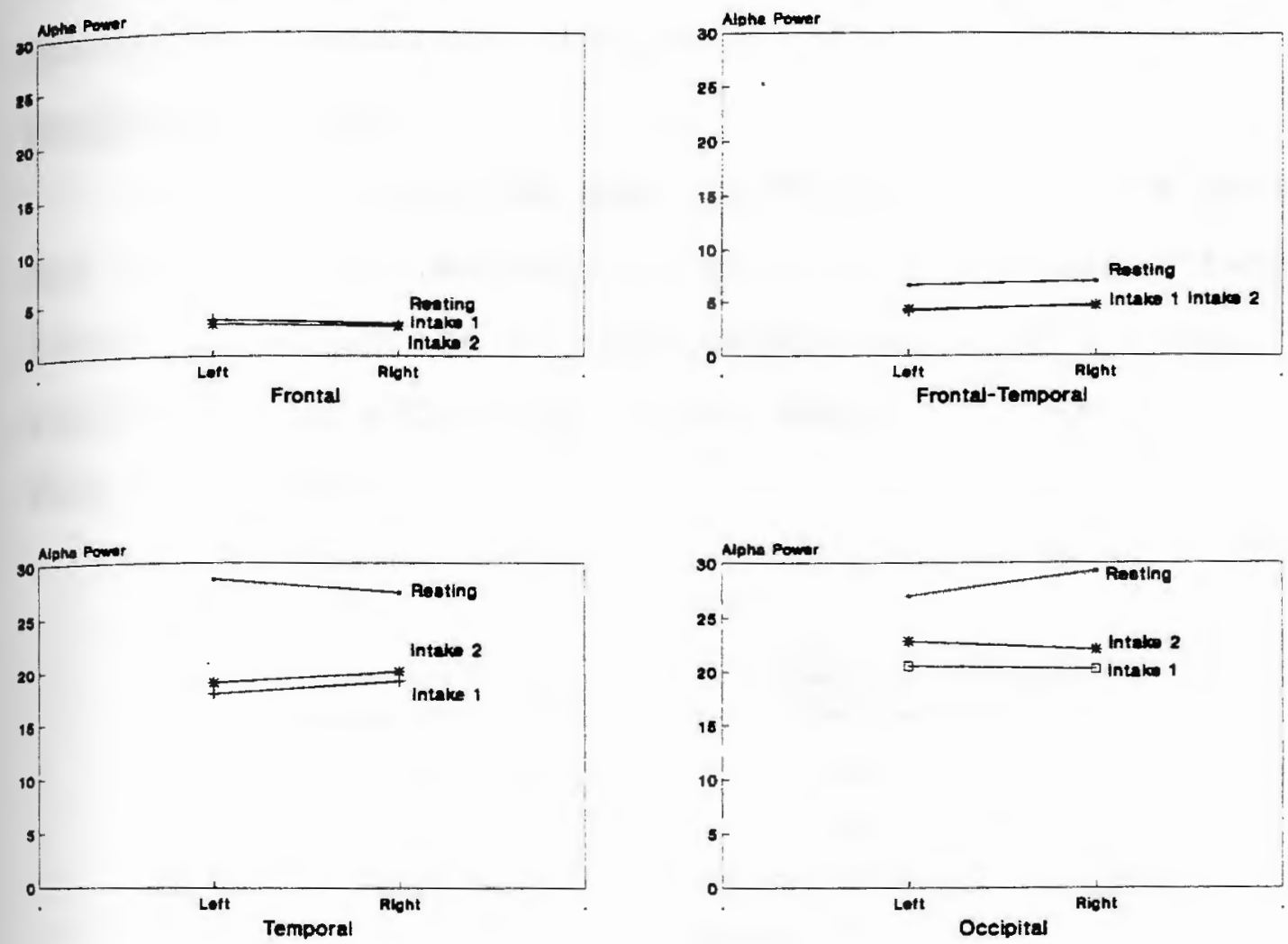

Prediction \#2: Simple contrasts at the temporaloccipital region showed that subjects exhibited significantly more alpha power in both the left, $F(1,86)$ $=4.18, p<.05$, and the right, $F(1,86)=9.7, p<.05$, hemispheres while resting than while performing the attention tasks, with no significant differences between intake 1 and intake 2 . 
Prediction \#3: Simple interaction effects tests at the frontal region failed to show any significant differences between the cerebral hemispheres during resting, intake 1 , or intake 2 .

\section{Performance Data}

Table 3 shows the mean performance (i.e. of correct) and standard deviations for males and females during intake 1 and intake 2. The performance data were analyzed with a two-way, mixed ANOVA with Gender and Task as factors.

Table 3

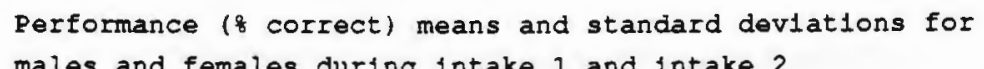
males and females during intake 1 and intake 2

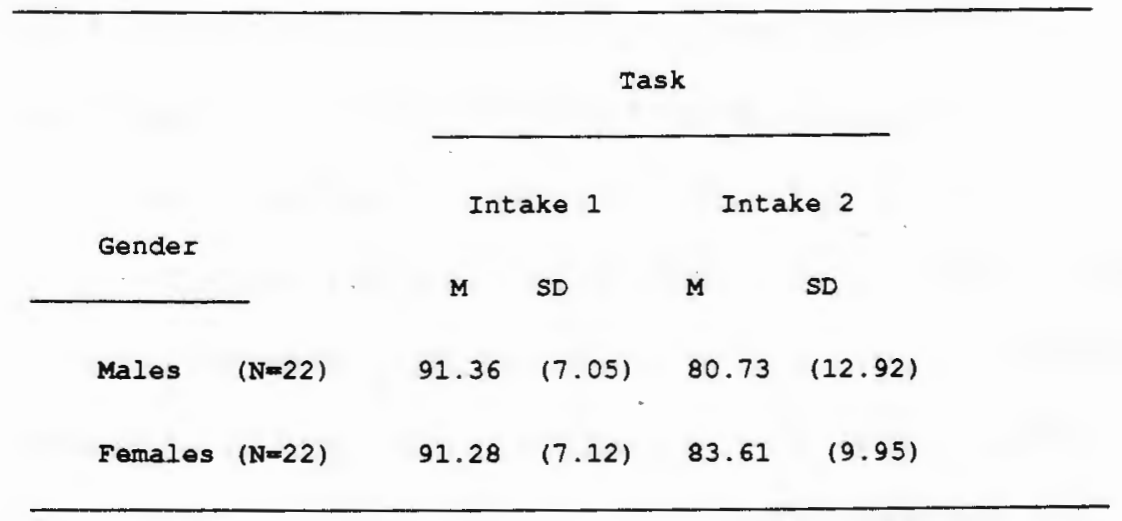

Prediction \#4: A significant main effect for CPT performance was obtained for Task with subjects scoring higher in intake $1(M=91.32)$ than in intake 2 $(M=82.17), F(1,42)=39.82, p<.01$, (see Appendix $F)$. 


\section{Discussion}

\section{PREDICTION ONE}

The results of this study do not support the hypothesis that males and females exhibit dissimilar activation of their cerebral hemispheres while performing a vigilance task. These results are consistent with those of Shepherd (1982). Nevertheless, before any conclusions can be put forth, other possible explanations for the observed result must be systematically explored.

One possible explanation may be that there are actual structural brain differences (Trotman \& Hammond, 1979) and/or processing strategy differences between males and females, but that the measure used in this present experiment was too insensitive to detect these differences. Despite the plausibility of this explanation, previous researchers have reported finding significantly different patterns of activation between males and females using EEG (Davidson et al., 1976; Earle \& Pikus, 1982; Glass et al., 1984; Ray et al., 1976; Trotman \& Hammond, 1979; Wogan et al., 1979). Hence, this explanation appears quite unlikely.

Another explanation might be that the measures were taken from the wrong locations on the head (i.e. regions of the cortex that are not gender specific). Indeed, this explanation might be an appropriate one if it were not for the fact that of those EEG studies that did 
report finding significant gender differences (Davidson et al., 1976; Earle \& Pikus, 1982; Glass et al., 1984; Ray et al., 1976; Trotman \& Hammond, 1979; wogan et al., 1979), only two included head locations not represented in the present study (Earle \& Pikus, 1982; Glass et al., 1984). Similarly, of the EEG studies that had reported finding no significant gender differences, all included head locations not included in the present study (Davidson et al., 1976; Galin et al., 1982; Harter et al., 1990; Shepherd, 1982). Consequently, this explanation appears insufficient also.

A third possible explanation may lie with the sample size chosen for this present experiment. A post hoc analysis of power indicated that with a sample of 22 males and 22 females and a treatment effect of 377.52 (Ssgender) the present experiment only possessed a power level of .35 . However, of those studies that did report finding gender differences, five of six studies used sample sizes either at or below 9 (i.e. nine males and eight females) (Davidson et al., 1976; Earle \& Pikus, 1982; Ray et al., 1976; Trotman \& Hammond, 1979; Wogan et al., 1979). As a result, either the error variances of these studies were extremely small, or their between groups effect sizes were extremely large. Unfortunately, such statistics were not made available by the authors.

A fourth, more plausible explanation might be that 
males and females possess similar brain structures, but that they utilize different cognitive strategies when faced with an elaborate task. In previous studies, subjects showed EEG differences only when they were engaged in complex spatial or verbal tasks. In those studies alpha asymmetries were greater for males than for females. However, when tasks used are complex they conceivably contain so many different cognitive components that any one strategy, from an array of many, may enable a subject to satisfactorily complete a given task. In the present investigation a continuous performance task (vigilance task) was chosen in order to control for this source of variability. It is being assumed that there is a restricted range of cognitive strategies that can be employed during an accurate performance of this task. A previous study (Shepherd, 1982) in which EEG was observed during a continuous performance task also failed to find significant gender differences, though that study only involved two bilateral electrode sites (O1-P3 and O2-P4).

If we were to assume that any hard-wiring difference between the male and the female brain would result in different patterns of activation for males and females, even when subjects performed a fundamental cognitive task such as a continuous performance task, it is reasonable to assume that previously found gender differences may be due to processing strategy 
differences only. Perhaps males and females possess the same hard wiring, but they utilize different, genderstereotyped processing strategies while performing a complex task (verbal and spatial tasks), but not when they are required to perform a simpler cognitive task (vigilance task). This difference in processing strategy might very well produce a corresponding difference in measured EEG. In fact, wogan et al. (1979) suggested that subjective reports of the strategies used by subjects might help to clarify the relationship between EEG and behavior, especially when subjects perform some of the more cognitively complex verbal and spatial tasks (p.223).

To conclude, these results do not lend support to the hypothesis that previous observations of EEG gender differences during task performance are related to differences in brain organization. The alternative hypothesis which states that observed gender differences are related to differences in the processing strategies of males and females appears to enjoy greater support.

\section{PREDICTION TWO}

The results of this study support the hypothesis that subjects exhibit an overall decrease in alpha power during the continuous performance task, relative to the resting condition. However, this phenomenon was limited to the left and right temporal-occipital regions. The prediction, which went a step further by predicting 
significant alpha-power differences between intakes 1 and 2, was not borne out by these results. These results are consistent with the previously documented and conventionally accepted relationship between mental effort and alpha power suppression (Marquis et al., 1984; Pollen \& Trachtenberg, 1972). Pollen and Trachtenberg (1972) reported that when subjects perform progressively more difficult mental tasks that there was a corresponding decrease of alpha power.

In the present investigation, alpha power in the left and the right temporal-occipital regions were the greatest during the resting condition and the lowest during the continuous performance task (i.e. intakes 1 and 2$)$. In the resting condition subjects were asked to sit still and relax their minds, hence their alpha power was greatest during this period. During the continuous performance task subjects were instructed to concentrate on a series of auditorily presented letters and to indicate when the same letter was presented consecutively (i.e. a target). The result was a corresponding low level of alpha power in the temporaloccipital regions.

Although these findings may appear straightforward and are intuitively appealing, there exists a flaw inherent in any comparison involving a resting condition. Unlike a continuous performance task, a resting condition lacks a behavioral measure of a 
subject's underlying cognitive state. In the present experiment, a subject's level of attention was operationally defined as the subject's performance on the continuous performance task. Hence, if a subject was not attentive while performing the continuous performance task, for whatever reason (e.g. thinking about sex, thinking about an argument the night before, etc...), that subject might have performed differently. In a resting condition, each subject, although instructed otherwise, is free to process different types of information. In addition, because these cognitions are not measured behaviorally, they cannot be controlled. Therefore, it is impossible to attribute observed differences in brain activity to assumed differences in cognitions. Indeed, none of the attention studies explored for this present study actually made statistical comparisons with a resting condition. The most appropriate comparisons would be between conditions that are identical in all, but one, verifiably controlled way.

In the present experiment EEG was measured at various points during the CPT (intakes 1 and 2 ). The two intakes were identical in every way except the subject's level of attention. However, no significant EEG differences between the two conditions were found. This might not have been the finding, however, if additional EEG data were recorded 
beyond the eight minute mark, allowing a greater separation between attention levels. Future studies of attention, conducted by the lab, should extend the duration of the continuous performance task, in order to provide a intelligible understanding of the relationship between attention, the brain, and performance.

\section{PREDICTION THREE}

The results of the present experiment do not support the hypothesis that the right, frontal region contains an attention mechanism specific to the maintenance of vigilance. There were no significant differences in alpha power between the left and the right frontal regions during the continuous performance task. These results are consistent with those of Heilman and Van Den Abell (1980) and Ray and Cole (1985) (EEG studies).

In the experiment conducted by Heilman and Van Den Abell, subjects performed a visual attention task which required them to signal the presence of a target. In the Ray and Cole study, subjects performed several intake and rejection tasks possessing both verbal and spatial components. Though vastly different tasks were used by the two studies, no significant alpha power differences between the frontal regions were reported. The remaining EEG studies failed to sample EEG from the frontal leads.

The two remaining brain imaging studies $(\mathrm{CBF}$ and 
$\mathrm{PET}$ ), however, did implicate the right, frontal region in attention (Haier et al., 1988; Roland, 1982). One possible explanation for the disparity between the present investigation's results and those results of Haier et al. and Roland may be that EEG is less sensitive than either PET or CBF. Indeed, because EEG is actually the summation of electrical activity throughout the brain, this explanation seems quite reasonable. Regardless of this explanation's plausibility, more $\mathrm{PET}$ and $\mathrm{CBF}$ research, using larger sample sizes, will be required to conclude that EEG is too insensitive for the investigation of the attention phenomenon.

\section{PREDICTION FOUR}

The results of the present experiment lend support to the hypothesis that the longer a subject is forced to be attentive, the greater the loss of attention. In the present study, subject performance was significantly better during intake 1 than it was in intake 2. The primary importance of this finding is simply to demonstrate that the task manipulation did work. Hence, the attention level of subjects, as operationally defined in the present experiment, did decline. However, there were no corresponding significant alpha power differences in any of the eight recording regions. 


\title{
Appendix A
}

\author{
Eectrode plocement.
}

CHANNELS
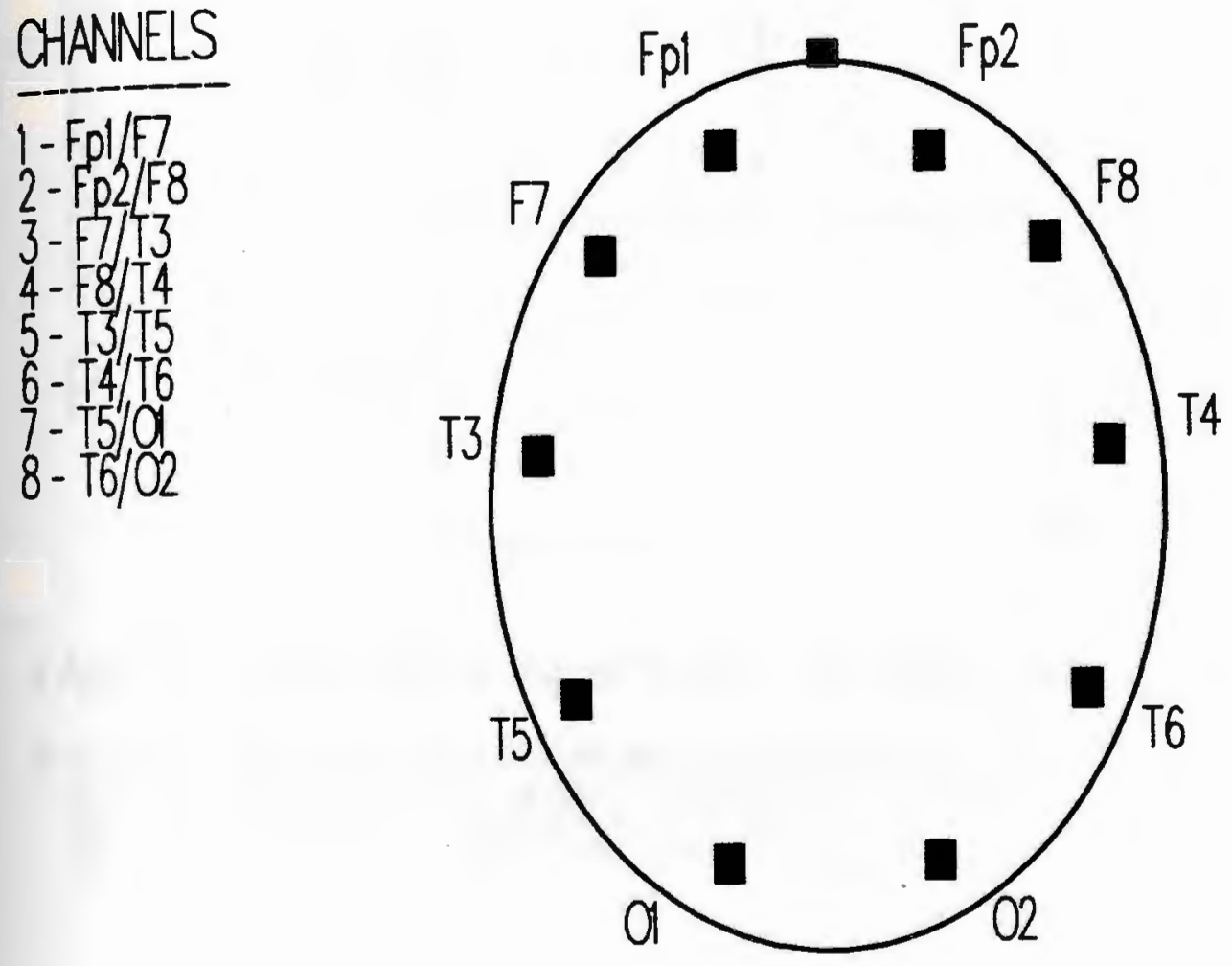


\section{Appendix B}

\section{RESTING * INTAKE I * INTAKE 2}

Time

$G$
$E$
$N$
$D$
$E$
$R$

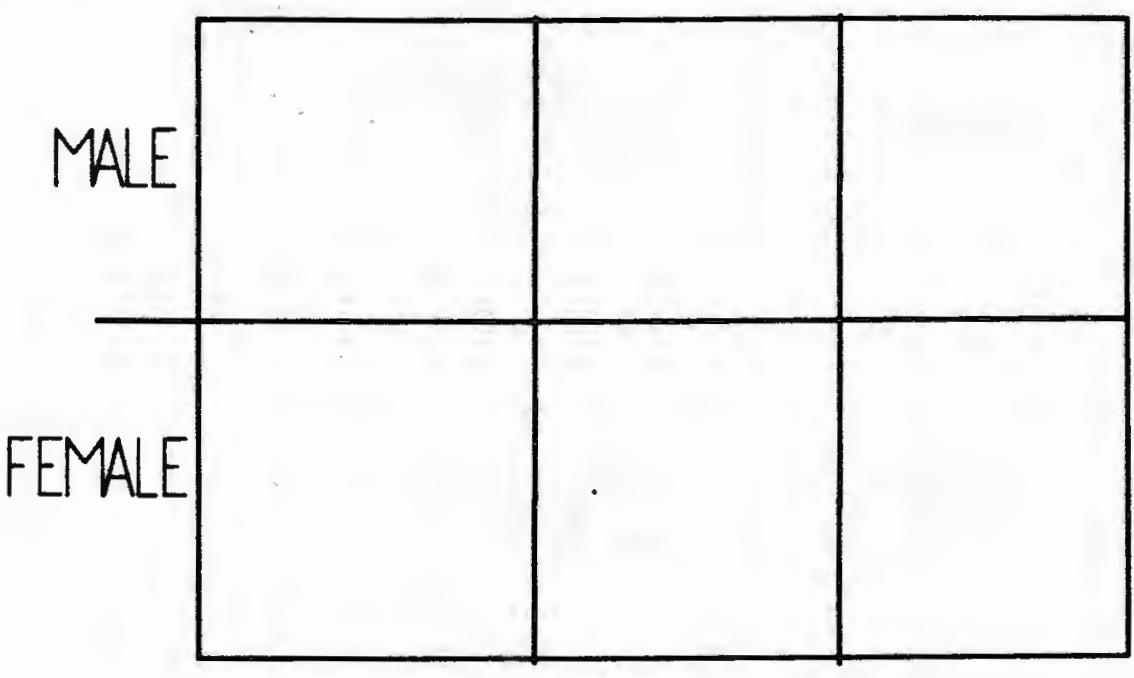

* Approximdely two minutes elopsed between Restingand intake 1.

* A six minute period occurred between the onset of the two

EEG periods, intake l ond Intake 2 subjects performed the

CPT losk continuously throughout this whole period 


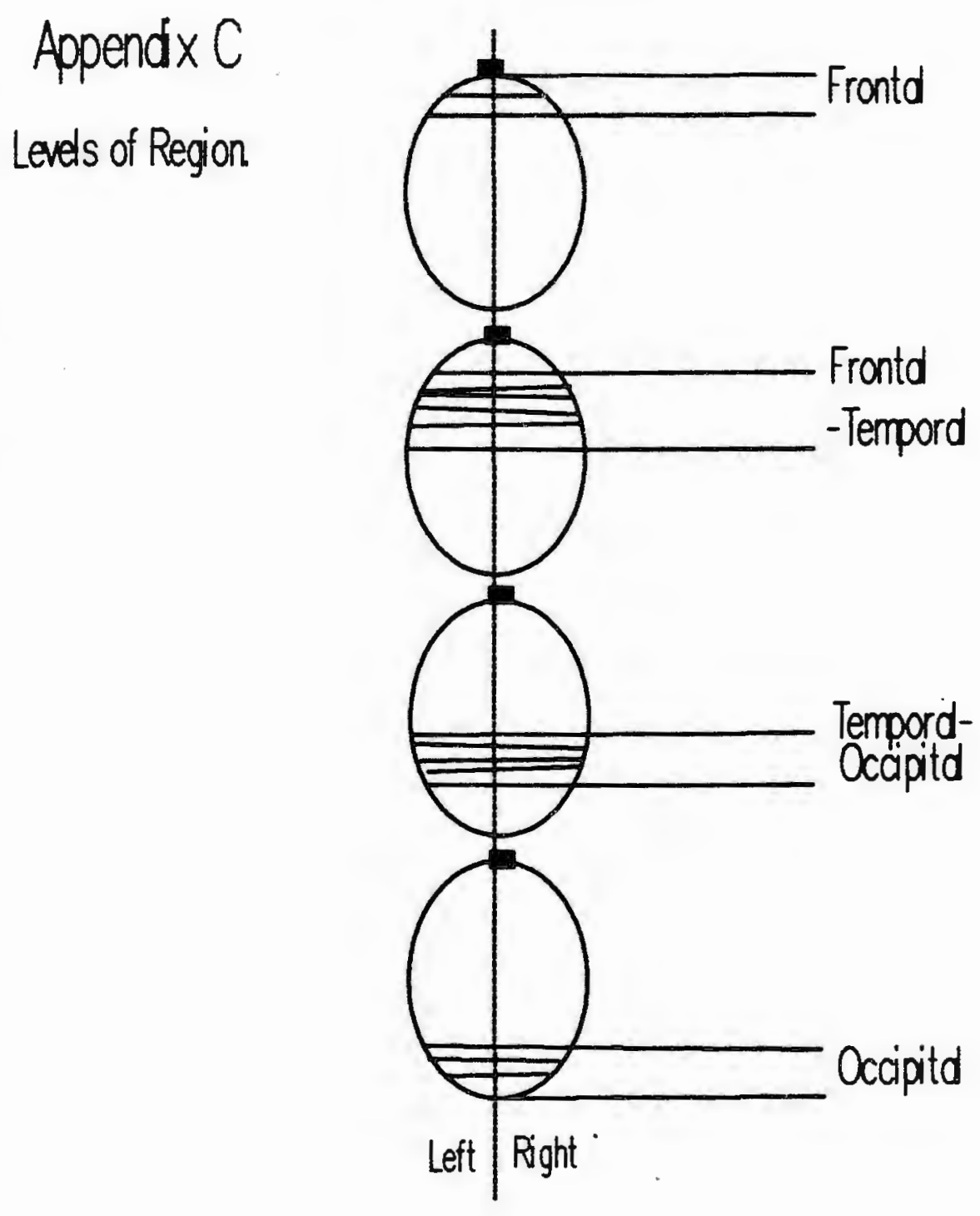


Append1x D

ANOVA source table for the four-way mixed design

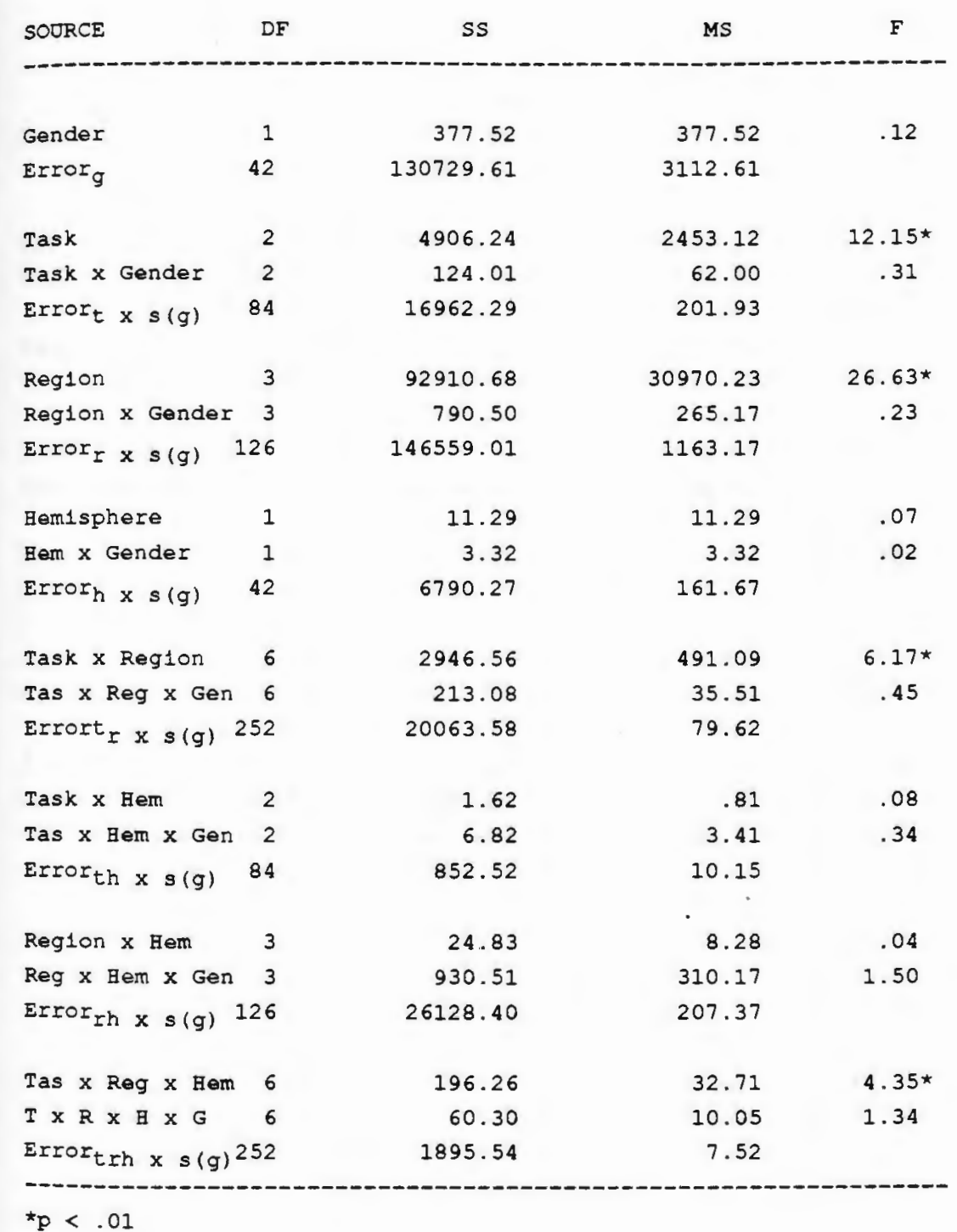


Appendix E

ANOVA source table for the three-way repeated measures design

\begin{tabular}{|c|c|c|c|c|}
\hline SOURCE & $D F$ & SS & MS & $F$ \\
\hline Task & 2 & 4906.24 & 2453.12 & $12.35 *$ \\
\hline Error $_{t}$ & 86 & 17086.30 & 198.68 & \\
\hline Region & 3 & 92910.68 & 30970.23 & $27.11 *$ \\
\hline Error I & 129 & 147354.51 & 1142.28 & \\
\hline Eemisphere & 1 & 11.30 & 11.30 & .07 \\
\hline Error & 43 & 6793.60 & 158.00 & \\
\hline Task $x$ Region & 6 & 2946.56 & 491.10 & $6.25 *$ \\
\hline ErrortI $_{\text {tI }}$ & 258 & 20276.67 & 78.60 & \\
\hline Task $\mathrm{x}$ Eem & 2 & 1.62 & .81 & .08 \\
\hline Errorth & 86 & 859.34 & 9.99 & \\
\hline Region $x$ Hem & 3 & 24.83 & 8.28 & .04 \\
\hline Error & 129 & 27058.91 & 209.76 & \\
\hline$T \times R \times B$ & 6 & 196.26 & 32.71 & $4.31 \star$ \\
\hline Errortrh & 258 & 1955.84 & 7.60 & \\
\hline
\end{tabular}




\section{Append1x $F$}

ANOVB source table for the two-way mixed design with performance

as the dependent measure

\begin{tabular}{lrrrr} 
SOURCE & DF & \multicolumn{1}{c}{ SS } & . MS & F \\
Gender & 1 & 43.28 & 43.28 & .32 \\
Errorg & 42 & 5747.90 & 136.85 & \\
Task & 1 & 1841.50 & 1841.50 & $39.82 \star$ \\
Task $x$ Gender & 1 & 47.96 & 47.96 & 1.04 \\
Error & 42 & 1942.12 & 46.24 &
\end{tabular}

$\star_{p}<.01$ 


\section{Bibliography}

Bear, D., Schiff, D., Saver, J., Greenberg, M., \& Freeman, R. (1986). Quantitative analysis of cerebral asymmetries. Archives of Neurology, $43,598-603$.

Berfield, K. A., Ray, W. J., \& Newcombe, N. (1986).

Sex role and spatial ability: An EEG study. Neuropsychologia, 24,731-735.

Bornstein, R. (1984). Unilateral Lesions and the Wechsler Adult Intelligence Scale-revised: No sex differences. Journal of Consulting and Clinical Psychology, 52, 604-608.

Bornstein, R., \& Matarazzo, J. (1982). Wechsler VIQ versus $P I Q$ differences in cerebral dysfunction: A literature review with emphasis on sex differences. Journal of Clinical Neuropsychology, 4 $319-334$.

Chui, H. C., \& Damasio, A. R. (1980). Human cerebral asymmetries evaluated by computed tomography. Journal of Neurology, Neurosurgery, and Psychiatry, 43, 873-878.

Davidson, R. J., Schwartz, G. E., Pugash, E., \& Bromfield, E. (1976). Sex differences in patterns of EEG asymmetry. Biological Psychology, 
$4,119-138$

Duffy, F. H., Albert, M. S., MceAnulty, G., \& Garvey, A. J. (1984). Age-related differences in brain electrical activity of healthy subjects. Annals of Neurology, 16, 430-438.

Edwards, S., Ellams, J., \& Thompson, J. (1976).

Language and intelligence in aphasia: Are they related? British Journal of Disorders in Communication, 11, 83-94.

Earle, J. B. B., \& Pikus, A. A. (1982). The effect of sex and task difficulty on eeg alpha activity in association with arithmetic. Biological Psychology, $15,1-14$.

Eriedland, J., \& Kershner, J. (1986). Sex linked lateralization central processor for hierarchically-structured material? Evidence from Broca's aphasia. Neurophychologia, 24, 411-415.

Galin, D., Ornstein, R., Herron, J., \& Johnstone, J. (1982). Sex and handedness differences in eeg measures of hemispheric specialization. Brain and Language, 16, 19-55.

Glass, A., Butler, S. R., \& Carter, J. C. (1984). Hemispheric asymmetry of eeg alpha activation: Effects of gender and familial handedness. 
Biological Psychology, 19, 169-187.

Haier, R. J., Siegel, B. V., Neuchterlein, K. H., Hazlett, E., Wu, J.C., Paek, J., Browning, H. I., \& Buchsbaum, M. S. (1988). Cortical glucose metabolic rate correlates of abstract reasoning and attention studied with positron emission tomography. Intelligence, 12, 199-217.

Harter, M. R., Miller, S. L., Price, N. J., LaLonde, M. E., \& Keyes, A. I. (1990). Neural processes involved in directing attention. Journal of Cognitive Neuroscience, 1, 223-237.

Heilman, K. M., \& Van Den Abell, T. (1978). Right hemispheric dominance for mediating cerebral attention. Neuropsychologia, 17, 315-321.

Heilman, K. M., \& Van Den Abell, T. (1980). Right hemisphere dominance for a.ttention: The mechanism underlying hemispheric asymmetries of inattention (neglect). Neurology, 30, 327-330.

Herring, S., \& Reitan, R. (1986). Sex similarities in verbal and performance IQ deficits following unilateral cerebral lesions. Journal of Consulting and Clinical Psychology, 54, 537-541.

Inglis, J., \& Lawson, J. (1981). Sex differences in the 
effects of unilateral brain damage on intelligence. Science, 212, 693-695.

Inglis, J., \& Lawson, J. (1982). A meta-analysis of sex differences in the effects of unilateral brain damage on intelligence test results. Canadian Journal of Psychology, 36, 670-683.

Jasper, H. H. (1958). Report of the committee on methods of clinical examination in electroencephalography. Electroencephalography and Clinical Neurophysiology, 10, 370-375.

Koff, E., Naeser, M. A., Pieniadz, J. M., Eoundas, A. L., \& Levine, H. L. (1986). Computed tomographic scan hemispheric asymmetries in right- and lefthanded male and female subjects. Archives of Neurology, 43, 487-491.

Lacoste-Utamsing, C. D., \& Holloway, R. L. (1982). Sexual dimorphism in the human corpus callosum. Science, 216, 1431-1432.

Lacoste, M. D., \& Woodward, D. J. (1988). The corpus in nonhuman primates. Brain, Behavior and Evolution, 31, 318-323.

Lansdell, H. (1961). The effect of neurosurgery on a test of proverbs. American Psychologist, 16, 448 . 
Lansdell, H. (1962). A sex difference in the effect of temporal lobe neurosurgery on design preference. Nature, 194, 852-854.

Iansdell, H. (1968a). Effect of temporal lobe ablations on two lateralized deficits. Physiology and Behavior, 3, 271-273.

Iansdell, H. (1968b). The use of factor scores from the wechsler-Bellevue scale of Intelligence in assessing patients with temporal lobe removals. Cortex, 4, 257-268.

Lansdell, H. (1973). Effect of neurosurgery on the ability to identify popular word associations. Journal of Abnormal Psychology, 81, 255-258.

Lewis, R. S., \& Kamptner, N. L. (1987). Sex differences in spatial task performance of patients with and without unilateral cerebral lesions. Brain and Cognition, 6, 142-152.

Marquis, F. A., Glass, A. \& Corlett, E. N. (1984). Speed of work and EEG asymmetry. Biological Psychology, 19, 205-211.

McGlone, J. (1978). Sex differences in functional brain asymmetry. Cortex, 14, 122-128.

McGlone, J. (1980). Sex differences in human brain 
asymmetry: a critical survey. The behavioral and Brain Sciences, 3, 215-263.

oppenheim, J. S., Lee, B. C., Nass, R., \& Gazzaniga, M.

S. (1987). No sex-related differences in human

corpus callosum based on Magnetic Resonance

Imagery. Annals of Neurology, 21, 604-606.

Pollen, D. A., \& Trachtenberg, M. C. (1972). Some problems of occipital alpha block in man. Brain Research, 41, 303-314.

Rapcsak, S. Z., Verfaellie, M., Fleet, S., \& Heilman, K. M. (1989). Selective attention in hemispatial neglect. Archives of Neurology, 46, 178-182.

Ray, W. J., Morell, M., \& Frediani, A. W. (1976). Sex differences and lateral specialization of hemispheric functioning. Neuropsychologia, $14,391-394$.

Ray, W. J., \& Cole, H. W. (1985). EEG alpha activity reflects attentional demands, and beta activity reflects emotional and cognitive processes. Science, 228, 750-752.

Roland, P. E. (1982). Cortical regulation of selective attention in man. A regional cerebral blood flow study. Annals of Neurophysiology, 48, 1059-1078. 
Salmaso, D. \& Denes, G. (1982). Role of the frontal lobes on an attention task: A signal detection analysis. Perceptual and Motor Skills, 54, 1147-1150.

Shepherd, R. (1982). EEG correlates of sustained attention: Hemispheric and sex differences. Current-Psychological-Research, 2, 1-19.

Snow, W., \& Sheese, S. (1985). Lateralized brain damage, intelligence and memory: A failure to find sex differences. Journal of Consulting and Clinical Psychology, 53, 940-941.

Sundet, K. (1986). Sex differences in cognitive impairment following unilateral brain damage. Journal of Clinical and Experimental Neuropsychology, 8, 51-61.

Trotman, S. C. A., \& Hammond, G. R. (1979). Sex differences in task-dependent EEG asymmetry. Psychophysiology, 16, 429-431.

Wada, J. A., Clarke, R., \& Hamm, A. (1975) Cerebral hemispheric asymmetry in humans. Archives of Neurology, 32, 239-246.

Witelson, S. F. (1985). The brain connection: The corpus callosum is larger in left-handers. Science, 229, 665-667. 
Wogan, M., Kaplan, C. D., Moore, S. F., Epro, R., \& Harner, R. - (1979). Sex differences and task effects in lateralization of eeg-alpha. International Journal of Neurosciences, 8, 219-223.

Yeo, R., Turkheimer, E., \& Bigler, E. D. (1984). The influence of sex and age on unilateral cerebral lesion sequelae. International Journal of Neurosciences, 24, 299-301.

Yeo, R., Turkheimer, E., Raz, N., \& Bigler, E. D. (1987). Volumetric asymmetries of the human brain: Intellectual correlates. Brain and Cognition, 6, 15-23. 